\title{
A prognostic model for the combined analysis of gene expression profiling in hepatocellular carcinoma
}

\author{
Zee Chung Ying Benny ${ }^{1, ~}$, Lee Jock Wai Jack ${ }^{1}$, Wong Nathalie ${ }^{2}$, Yeo Winnie ${ }^{3}$, Lai Bo San Paul ${ }^{4}$, Mok Shu Kam Tony ${ }^{3}$, \\ Chan Tak Cheung Anthony ${ }^{3}$
}

${ }^{1}$ Centre for Clinical Trials, School of Public Health; ${ }^{2}$ Department of Anatomic and Cellular Pathology; ${ }^{3}$ Department of Clinical Oncology; ${ }^{4}$ Department of Surgery, Chinese University of Hong Kong, Shatin, Hong Kong SAR; Benny Zee* -E-mail: bzee@cct.cuhk.edu.hk; *Corresponding Author

received February 26, 2008; revised March 07, 2008; accepted May 14, 2008; published July 14, 2008

\begin{abstract}
:
Microarray techniques using cDNA array and comparative genomic hybridization (CGH) have been developed for several discovery applications. They are frequently applied for the prediction and diagnosis of cancer in recent years. Many studies have shown that integrating genomic data from different sources may increase the reliability of gene expression analysis results in understanding cancer progression. Therefore, developing a good prognostic model dealing simultaneously with different types of dataset is important. The challenge with these types of data is high background noise. We describe an analytical two-stage framework with a multi-parallel data analysis method named wavelet-based generalized singular value decomposition and shaving method (WGSVD-shaving). This method is proposed for de-noising and dimension-reduction during early stage prognosis modeling. We also applied a supervised gene clustering technique with penalized logistic regression with Cox-model on an integrated data. We show the accuracy of the method using a simulated dataset with a case study on Hepatocelluar Carcinoma (HCC) cDNA and CGH data. The method shows improved results from GSVD-shaving and has application in the discovery of candidate genes associated with cancer.
\end{abstract}

Keywords: wavelets; generalized singular decomposition; Cox-model; HCC

\section{Background:}

Hepatocellular carcinoma (HCC) is one of the major malignancies worldwide and more than half of the cases occur in China. The course of the disease (HCC) does not only vary depending on disease characteristics, but also the association between genotyperelated differences. There is a poor prognosis for HCC. DNA microarray technology has been proved to be an effective tool for prognosis and to identify genes that are potential therapeutic molecular targets [1-3]. The drawback of this type of data is the lack of potential power for detecting the regional impacts. Even if conventional CGH allows assessment of changes in chromosomal DNA sequence copy numbers across the genome and provide valuable information regarding genetic alternations in cancer [4-6].

The CGH cannot detect changes in small chromosomal regions. Researchers $[7,8]$ have developed methods to identify the regions 1q, 4q, 8q, 16q, 20q and Y, which show common alterations in the given HCC samples. Many of the related HCC studies have similar results but with different approaches. However, the analysis of locating genes with highest variation in copy number with the strongest correlation across all samples together with clinical factors is rarely addressed. It is known that integrating data from different sources such as DNA, gene and protein level data may increase the reliability of the analysis results. A combination of copy number data with gene expression data can address cases with increased or decreased copy number in gene over or under expression $[\mathbf{9}, \mathbf{1 0}]$.
Berger and colleagues applied a generalized singular valued decomposition with iterative shaving method (GSVD-shaving algorithm) to examine the patterns of expression which correlate to patterns of copy number on a global scale. They also examine the effects of including array CGH profiles in conjunction with expression profiles for variable selection in breast cancer data [11]. Attaining a suitable global scale is quite limited by the data type and there are noisy signals leaking into the transformed scaled data. This is true when the conventional CGH measurements have less size than gene expression measurements.

Here, we describe the construction of a two stage analytical framework. We propose a multi-parallel data analysis method, named wavelet-based generalized singular value decomposition and shaving method (WGSVD-shaving). Subsequently, we apply a supervised gene clustering with penalized logistic regression [13] with Cox-model method on the combination of clinical factors and reduced data from early stage to find prognostic indicators. We use the data from Berger and colleagues [11] for the analysis. We also conduct a case study on HCC with gene expression and CGH data. It should be noted that during the early stage we are describing a new data scale transformation method using wavelet coefficients from robust smoothing-cleaner wavelet approach [14]. In the HCC study, we apply the WGSVD-shaving for dimension reduction analysis of combined CGH and cDNA expression in 20 samples (including 9 short survivors and 11 long survivors). 


\section{www.bioinformation.net}

A penalized logistic regression with Cox model is used on retained genes with clinical data from HCC samples. Throughout the analysis, the comparison of WGSVD-shaving and GSVD-shaving method is used. Using the WGSVD-shaving method with the proper choice of projection angle $\theta$ in the GSVD, we are able to improve the results by using GSVD-shaving method. Moreover, the results have shown that the most frequent aberration of genomic material was gain of $1 \mathrm{q}$ and loss of $16 \mathrm{q}$. The impacted genes include $\mathrm{H} 20345$, R71531 and H20256. The major detected region is C8p23.1 and the detected clinical factor is histology differentiation (hdif). We used a diagnostic test of Receiver Operating Characteristics (ROC) curve is applied to verify these results.

The objective of this study is to develop a method to improve the GSVD-shaving [11-12] for de-noising by analyzing both gene expression and copy number data in conjunction to detect useful genes (and regions). We use these genes (regions) with clinical factors in cancer to find significant prognostic indicators. We are particularly interested in examining genes from the common region for increasing the reliability of the analysis results.

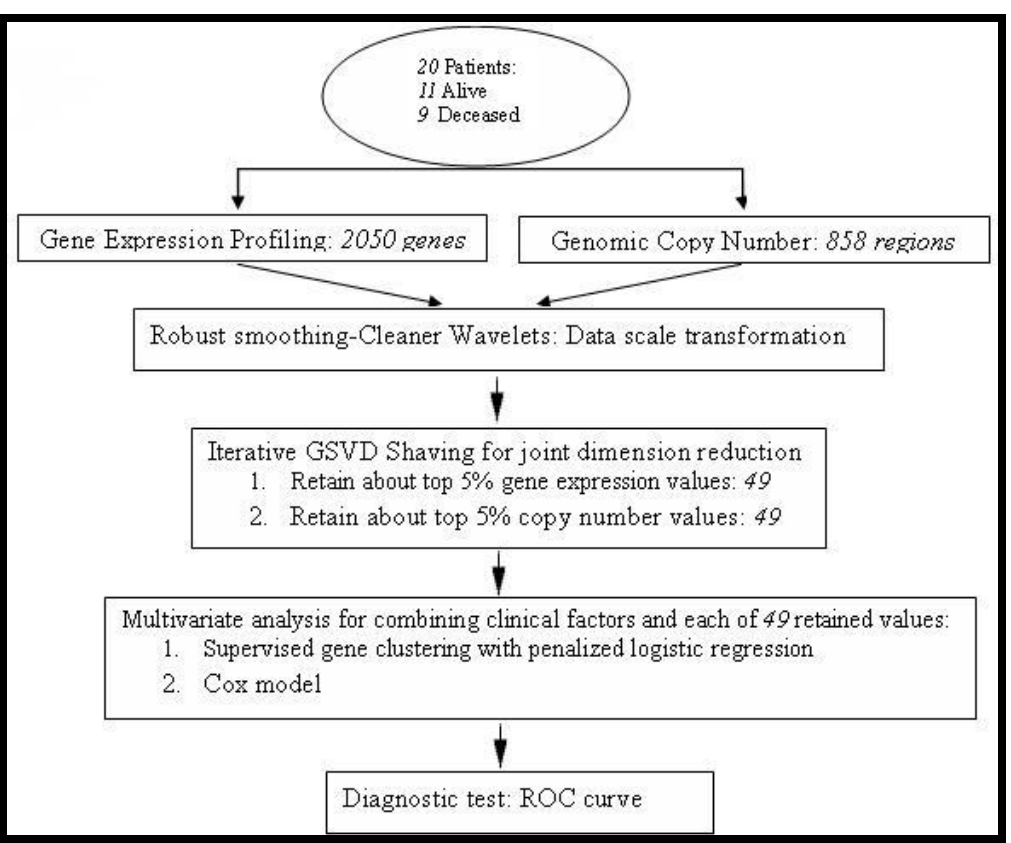

Figure1: The framework for prognosis analysis based on wavelet-based combined with the analysis of cDNA and CGH data.

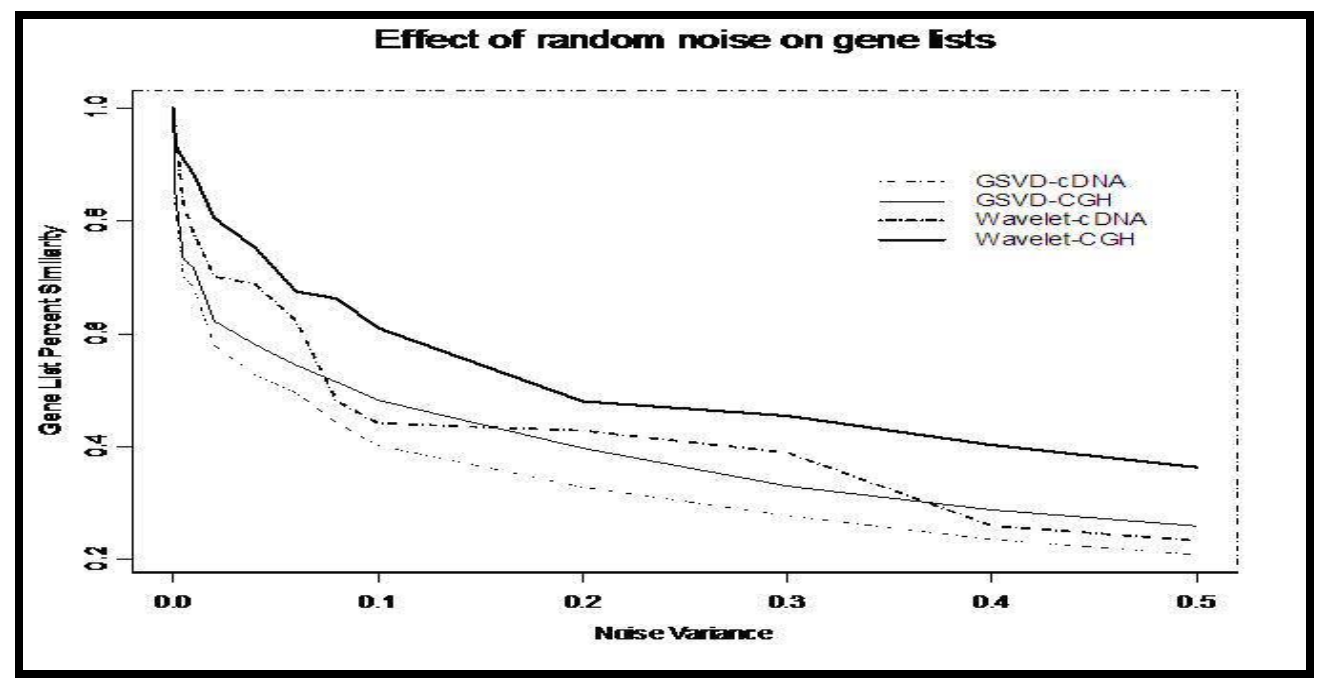

Figure 2: Effect of random noise on gene lists. We compared the effects of additive noise on cDNA and CGH data using both GSVDshaving and WGSVD-shaving algorithms.

ISSN 0973-2063 


\author{
Methodology: \\ Conventional CGH data for HCC \\ Conventional $\mathrm{CGH}$ analysis on surgical $\mathrm{HCC}$ is performed on the \\ 202 HCC samples. This is carried out according to our previously \\ described method by Wong and colleagues [15]. There are 20 \\ samples (patients) with 858 preprocessed CGH regions each used in \\ the analysis.
}

\section{cDNA gene expression profiling for $\mathrm{HCC}$}

Expression microarray study is done according to the protocol of UHN Microarray Centre [16]. We used the same sample set in CGH data but with 2050 preprocessed genes each. We describe here a framework of a prognostic analysis as shown in figure 1 for the HCC dataset. The detailed procedure of WGSVD-shaving algorithm is illustrated in supplementary material.

\section{Analysis:}

The analysis is divided into two stages as illustrated in Figure 1.

\section{Wavelet-based GSVD-Shaving method}

We integrated CGH copy number data with gene expression profiles for locating similar and dissimilar patterns of variation. We preprocessed and transformed the datasets by using wavelet transformation (RSCWT) since the similarity of data scales from two different types of data sets is essential. The detailed procedures are described in steps 1-6 (see supplementary materials). We applied (steps 7-13) the joint dimension reduction formulation to analyze gene expression and copy number alterations over experimental HCC datasets to extract results. We also adapted a simulation method from Berger and colleagues [11] to generate the random data sets and to provide a de-noising comparison between WGSVD-shaving and GSVD-shaving. The de-noising measurement is given in equation 1 (see supplementary materials). In step 2, we applied the robust smoother-cleaner wavelet transform (RSCWT) [14], a fast wavelet decomposition which is robust towards outliers. Smoother-cleaner wavelets behave like the classical L2 wavelet transform for Gaussian signals. However, it prevents outliers and outliers patched from leaking into the wavelet coefficients at coarse levels. We used the "b-spline" bi-orthogonal wavelets sine. It is a class of filters which satisfy both requirements: short filters can be used for decomposition and longer filters for reconstruction. The smoother-cleaner step is based on a median filter. We selected the median filters of length $\mathrm{L} \geq 2 \mathrm{M}+1$ for a low-pass wavelet filter of length $\mathrm{M}$ to avoid smearing for most types of wavelets and the usual value assigned to $L$ is 5 .

\section{Multivariate analysis for prognostics indicators}

We applied the penalized logistic regression method [13] to cluster the combined datasets (from early stage) and clinical factors. It is an algorithm that is based on penalized logistic regression analysis by using external information about response variables for clustering genes. The toolkit for the implementation of this method in $\mathrm{R}$ is Pelora. Then the multivariate Cox-model is applied to select those statistically significant prognostic indicators that are highly associated with HCC. The significant factors are finally verified by ROC curve analysis and with previously published data [7-8]. The ROC curve [17] by varying the parameter survival time is plotted for selected indicators and performance evaluation corresponding to WGSVD-shaving and GSVD-shaving is established.

\section{Discussion: \\ De-noising performance}

We used wavelet-based GSVD-Shaving method and GSVDShaving method to analyze the datasets which is an ensemble of 1000 expression and copy number data derived in a simulation study. Each set has $n=p=1500$ genes in 3 samples. We compared the two methods based on the percentage similarity (PS) index (see equation 1 in supplementary material). The simulated result also shows that analysis of 75 remaining genes in the dataset by adding random noise with repeating 1000 separate times for each variance level. Each set is in the ensemble of generated data and variance is incremented in the range 0 to 5 . The results from Figure 2 show that the range of cDNA in PS is from 1 decreasing to nearly 0.21 based on GSVD-shaving method. Simultaneously, the range of cDNA in PS based on wavelet is from 1 decreasing to about 0.23 . In array $\mathrm{CGH}$, the GSVD-shaving method gives 1 to 0.26 and the waveletbased approach the ranges from 1 down to 0.36 . The de-noising is consistently improved as variance is changed and in average about $8 \%$ greater accuracy is achieved than GSVD-shaving for cDNA data and $12 \%$ for array CGH data. It is observed that de-noising using wavelet is high.

\section{HCC data analysis}

We have CGH and cDNA expression data from $20 \mathrm{HCC}$ cell lines comprising 11 short survivors and 9 long survivors at median survival time of 1.96 years. On the basis of the described framework, we used both WGSVD-Shaving and GSVD-Shaving to analyze the HCC datasets for dimensionality reduction. We then applied penalized logistic regression to analyze these reduced HCC genomic data in addition to clinical information. We are interested in three angular distances $\theta \max , \theta \approx 0$ and $\theta \min$ and these distances explain biological insights from gene expression data analysis. In some genes with over-regulated state in expression and are associated with deleted region from copy number in parallel we examine angular distances of $\theta \max$ or $\theta$ min (in many cases they have similar results). Here we present the comparison between GSVD-shaving and wavelet GSVD shaving at $\theta \max$ (see Figure $3 \mathrm{a}$, Figure $3 \mathrm{~b}$ for cDNA reduced data and Figure $4 \mathrm{a}$, Figure $4 \mathrm{~b}$ for $\mathrm{CGH}$ reduced data). 


\section{Bioinformation}

\section{www.bioinformation.net}

\section{Hypothesis}

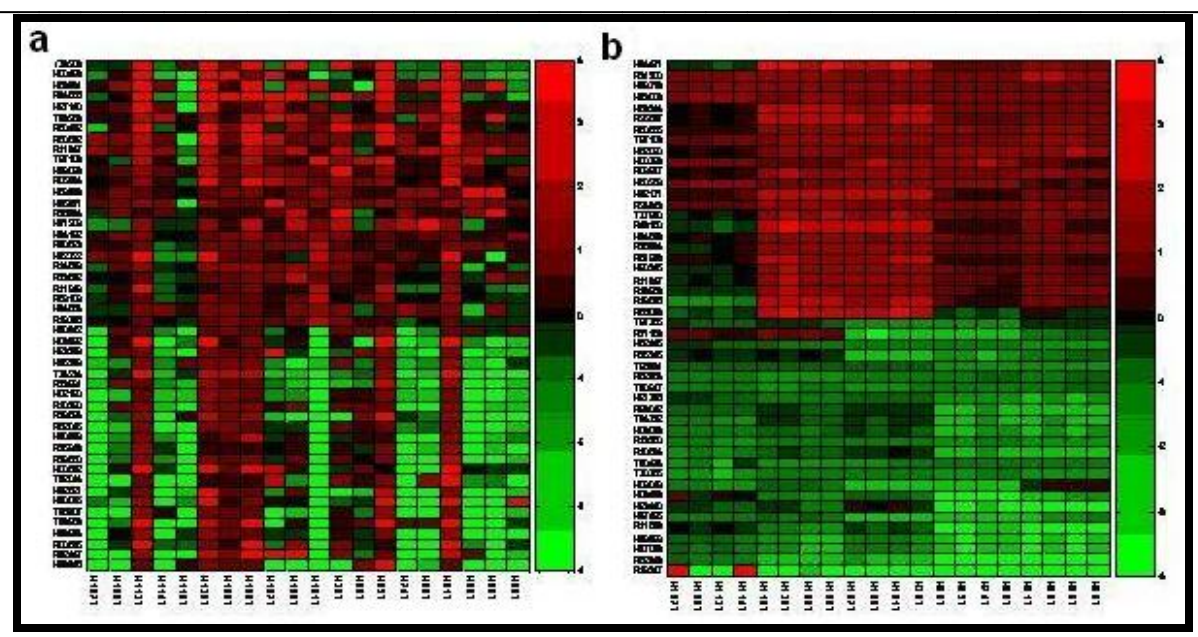

Figure 3: The retained top 49 highest variant genes display their expressions across $20 \mathrm{HCC}$ samples and the patterns show that the highest parallel contributions to the iterative projections after shaving out all other genes: (a) by GSVD-shaving approach, (b) by WGSVD-shaving approach.

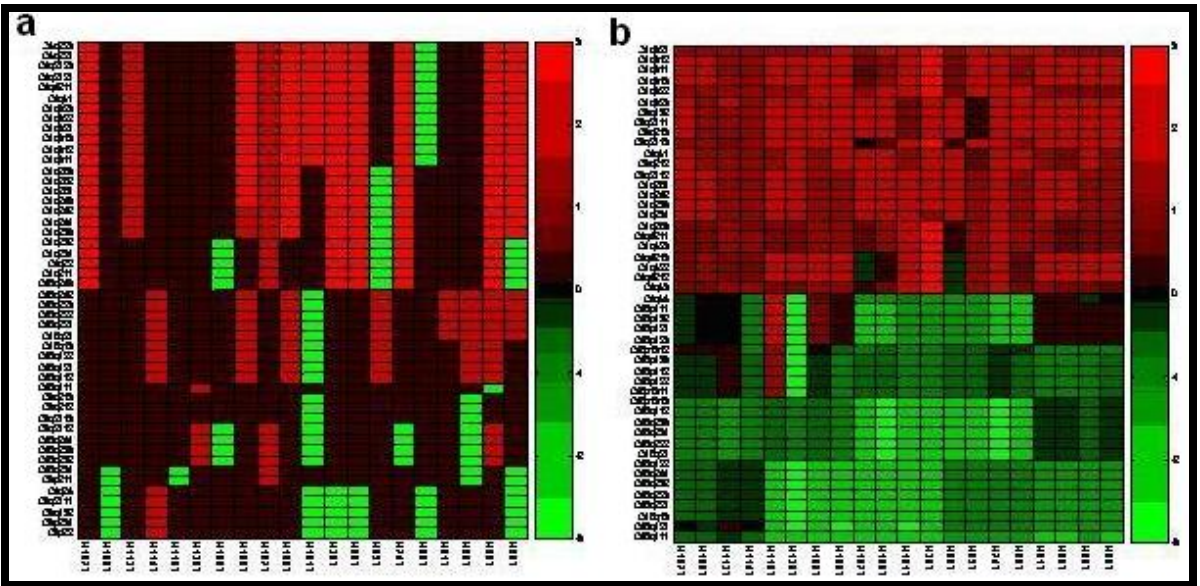

Figure 4: The retained top 49 highest variant genes display their copy number ratio (transformed) across 20 HCC samples and the patterns shows genes with highest variation in copy number and strongest correlation across all samples: (a) by GSVD-shaving approach, (b) by WGSVD-shaving approach.

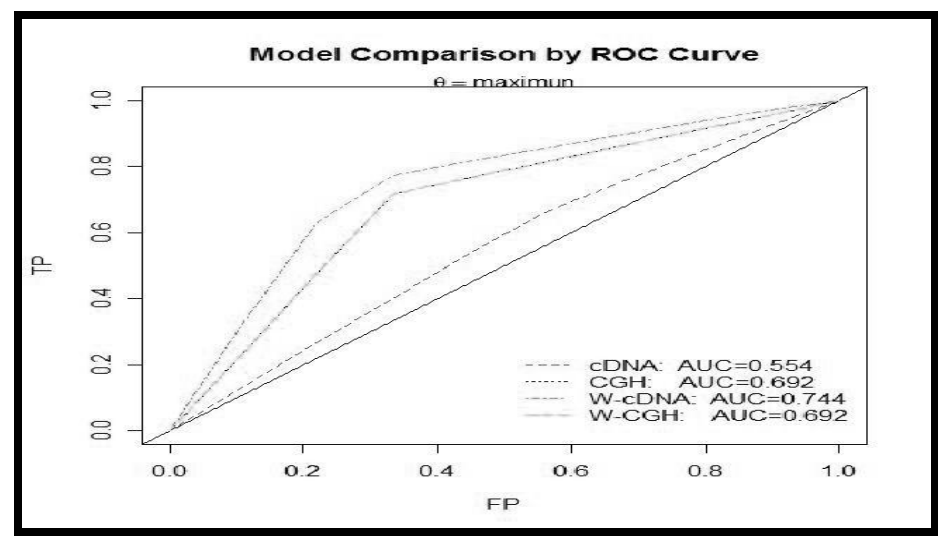

Figure 5: Comparison of results between GSVD and wavelet-scaled GSVD at $\theta$ max.

ISSN 0973-2063 


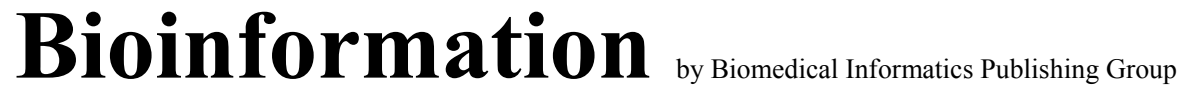

open access

\author{
www.bioinformation.net
}

Hypothesis

\begin{abstract}
We see that the overall patterns from WGSVD-shaving are more orderly distributed for classification in two interested states (underexpression/green and over-expression/red) on comparison to GSVD-shaving (Figures 3a and Figure 3b). The method described here show less noise. A similar observation is seen in Figure 4a and Figure $4 \mathrm{~b}$. The patterns in WGSVD-shaving are more ordered distributed for classification in two states (deletion/green and amplification/red) on comparison to GSVD-shaving. This indicates the analyzed data has less noise disturbances under the method. The ranked genes and regions selected are more ordered (classified) from any of three angular distances described in the method. We also applied penalized logistic regression to analyze both retained (reduced) genomic data supplemented with clinical information. The usual multivariate analysis of Cox-model is applied for the results obtained from penalized logistic regression and the results are shown in Figure 5.
\end{abstract}

The results of GSVD-shaving approach present three genes from cDNA data with respect to high-low risk (dead/alive) of patients T74214 ( $\mathrm{p}=0.0313), \mathrm{H} 43974(\mathrm{p}=0.0176), \mathrm{H} 08892(\mathrm{p}=0.0435)$ with no detection of clinical factors at $\theta$ max. The ROC curve analysis shows that the Area-Under-the-Curve (AUC) is 0.554. We obtained $\mathrm{H} 20345(\mathrm{p}=0.0070)$ and histology differentiation (hdif) $(\mathrm{p}=0.0237)$ using WGSVD-Shaving. The observed AUC is 0.744 and is much higher than GSVD shaving method. Both methods with Multivariate Cox Model show no significant regions (at 95\%CI) for $\mathrm{CGH}$ data in HCC. However, clinical factor "hdif" is detected by WGSVD-shaving. Other angular distances show similar results except for WGSVD-shaving which discovered the significant region C8p23.1. Thus the method described is useful for the discovery of candidate genes with associated regions in cancer.

\section{Conclusion:}

We developed a useful framework for cancer prognosis and described an effective combination genomic data analysis method named WGSVD-shaving. The method was first tested by a simulation study and then compared with the GSVD-shaving method with $8 \%$ and $12 \%$ higher de-noising (in accuracy) for cDNA and CGH data, respectively. Results show that performance by WGSVD-shaving is higher than GSVD-shaving. The methods were evaluated using ROC curve analysis and data show that
WGSVD-shaving has a higher probability to classify HCC with selected prognostic indicators than GSVD-shaving. The method can also pick genes and chromosome locations of biological significance with histological information.

\section{Acknowledgement:}

This study is funded by the Research Grant Council of Hong Kong \#CUHK4469/03M.

\section{References}

[01] J. Khan, et al., Nat Med., 7: 673 (2001) [PMID: 11385503]

[02] S. L. Pomeroy, et al., Nature, 415: 436 (2002) [PMID: 11807556]

[03] D. G. Beer, et al., Nat Med., 8: 816 (2002) [PMID: 12118244]

[04] O. P. Kallioniemi, et al., Gene Chromosomes Cancer, 10: 231 (1994) [PMID: 7522536]

[05] S. Knuutila, et al., Am. J. Pathol., 152: 1107 (1998) [PMID: 9588877]

[06] L. A. James, J Pathol., 187: 385 (1999)

[07] J. Joseph and A. Kyle, Genome Biology, 3: 12 (2002) [PMID: 12537564]

[08] S. J. Park, et al., Cancer Genet Cytogenet., 166: 56 (2006) [PMID: 16616112]

[09] J. R. Pollack, et al., Proc. Nat. Acad. Sci. USA, 99: 12963 (2002) [PMID: 12297621]

[10] S. Hautaniemi, et al., J. Franklin Inst., 341: 77 (2004)

[11] J. A. Berger, et al., IEEE Trans Computl Bio Bioinform., 3: 2 (2006) [PMID: 17048389]

[12] http://www.ece.ucsb.edu/pubs/ieee/

[13] D. Marcel and B. Peter, Report No.: 115, SfS Research, Zuerich (2003)

[14] A. G. Bruce, et al., In SPIE Proceedings: Wavelet Applications, Orlando, 325 (1994)

[15] P. Wang, et al., Biostatistics, 6: 45 (2005) [PMID: 15618527]

[16] http://www.uhnres.utoronto.ca/services/microarray/protoco 1s/

[17] P. J. Heagerty and Y. Zheng, Biometrics, 61: 92 (2003) [PMID: 15737082]

Edited by P. Kangueane

Citation: Benny et al., Bioinformation 2(9): 395-400 (2008)

License statement: This is an open-access article, which permits unrestricted use, distribution, and reproduction in any medium, for non-commercial purposes, provided the original author and source are credited. 


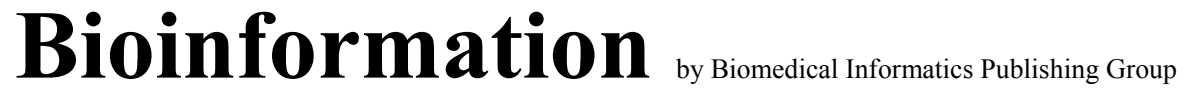

open access

\author{
www.bioinformation.net
}

Hypothesis

\section{Supplementary material}

\section{WGSVD-shaving Algorithm}

(1) Let $n>p$ for the given two block sub-matrices $R_{A} \in R^{n \times m}$ and $R_{B} \in R^{p \times m}$ that contain the cDNA and CGH data after preprocessing and normalization.

(2) Obtain wavelet coefficients by using robust smoothing-Cleaner Wavelets on cDNA and CGH values.

(3) Take mean values from above wavelet coefficients of cDNA and discard $n-p$ wavelet coefficients near zero. This will provide $n^{\prime}=p$.

(4) Rank-sort after-discarding wavelet coefficients of cDNA in a vector $w R_{A^{\prime}}^{\text {sort }} \in R^{\left(n^{\prime} \times m\right) \times 1}$ and rank-sort wavelet coefficients of $\mathrm{CGH}$ in a vector $w R_{B}^{\text {sort }} \in R^{(p \times m) \times 1}$.

(5) Obtain a polynomial fit $f$ with specified order for the data vector $w R_{A^{\prime}}^{\text {sort }}$, which is the best in the least-square sense.

(6) Transform each element in the wavelet coefficient matrix according to the equation $w R_{B^{\prime}}=f\left(w R_{B}\right)$ and form the matrix

$$
w R=\left[\begin{array}{c}
w R_{A^{\prime}} \\
w R_{B}
\end{array}\right] .
$$

(7) Compute U, V, X, $\Sigma$ A, and $\Sigma \mathrm{B}$ using GSVD.

(8) Analyze $\theta_{i}$ for $\mathrm{i}=1, \ldots, \mathrm{m}$ and select the direction of $\theta_{\min }, \theta_{\max }$, and $\theta_{i} \approx 0$.

(9) Project $w R$ onto the $k$ th column of $\mathrm{X}$ corresponding to $\theta_{k}$ as taken in Step 8.

(10) Retain the top $\eta=95$ percent of genes with the highest parallel contribution from $w R_{A^{\prime}}$ and $w R_{B}$

(11) Apply the gene shaving method and reform the matrix $w R$.

(12) Repeat Steps 7-11 if the number of genes is greater than or equal to the number of samples $\left(n^{\prime}+p \geq m\right)$.

(13) Analyze the top 5-10 percent highest variant genes through visualization and functional assessment.

\section{Equations:}

\section{Denoising measurement}

The computed gene list percentage similarity (PS) by counting the number of genes intersecting the list obtained using noisy data (ND) and the reference list obtained with original data $(\mathrm{OD})$.

The sum is divided by the number of total genes in the list (Tot):

$$
P S=\left(\frac{\# N D \cap \# O D}{\# T o t}\right) \times 100 \% \quad \rightarrow \quad
$$

